\title{
Die Bewegung der Blockfreien - einige bibliographische Notizen
}

\author{
Von Volker Matthies
}

\section{Blockf reiheit: Ein vernachlässigtes Forschungsfeld internationaler Beziehungen}

Im Jahre 1981 feierte die Bewegung der Blockfreien ihr zwanzigjähriges Bestehen. ${ }^{1}$ Sie erinnerte an die erste Konferenz der Staats- und Regierungschefs blockfreier Länder in Belgrad 1961 und würdigte die historische Rolle der Blockfreiheit als "unabhängige moralische und politische Kraft sowie als positiver und globaler Faktor des Friedens und der Unabhängigkeit in der Welt". Zugleich bekräftigten die Blockfreien ihren Willen zu verstärkter Solidarität und Zusammenarbeit untereinander, um angesichts des stagnierenden Nord-Süd-Dialoges und verschärfter Ost-West-Spannungen ihren besonderen Anliegen und Interessen im Nord-Süd- und Ost-West-Verhältnis mehr Nachdruck zu verleihen.

Lange Jahre hindurch blieb die Bewegung der Blockfreien von Politik, Publizistik und Wissenschaft vernachlässigt. Die Politik nahm die Blockfreien allenfalls als einen ziemlich unbedeutenden, wenngleich auch manchmal lästigen Irritationsfaktor der internationalen Beziehungen wahr, während die Publizistik, sofern sie sich des Themas überhaupt annahm, überwiegend polemisch und verzerrend über die Bewegung berichtete. Kaum jemals wurde das Phänomen der Blockfreiheit systematisch in das Studium Internationaler Beziehungen einbezogen, und auch die Friedensforschung ignorierte Blockfreiheit als einen möglichen Gegenstand ihres Interesses. ${ }^{2}$

Wenngleich die Bewegung der Blockfreien mittlerweile sowohl von der Politik als auch von der Forschung als ein relevanter und respektabler Faktor der internationalen Beziehungen erkannt und anerkannt worden ist, ${ }^{3}$ so muß der Stand der Forschung zur Blockfreiheit jedoch weiterhin als unbefriedigend bezeichnet werden. Scharfe, doch wohl berechtigte Kritik an der westlichen Forschung übte in diesem Zusammenhang der Inder Bandyopadhyaya:

". . . it is only the ethnocentric prejudice and intellectual myopia of the Western theorists which prevent them, with a few rare exceptions, from recognizing non-alignment as at

I Vgl. hierzu z. B. Volker Matthies, Von der Unmoral zur Respektabilität. Zwei Jahrzehnte Bewegung der Blockfreien (1961-1981), in: Vereinte Nationen 6/81, S. 183-188.

2 Dies kritisierte zu Recht Bojana Tadic, Peace Research and Non-Alignment, in: International Problems (Belgrad), 1972, S. $129 \mathrm{ff}$.

3 So befaßte sich z. B. der Weltkongreß der Political Science Association in Moskau 1979 erstmalig mit Fragen der Blockfreiheit. In der Bundesrepublik Deutschland kam es 1980 zur Gründung einer "Dokumentationsstelle Bewegung Blockfreier Staaten, e. V." die zum Stand vom 20. Juni 1981 eine Aufnahme ihres Bestandes vorlegte (Klaus Fritsche, Die Bewegung Blockfreier Staaten. Bestand der Dokumentationsstelle Bewegung Blockfreier Staaten, e. V., Dortmund). 
least one of the major models of the international system and giving it some space in their textbooks on international relations. " ${ }^{4}$

Um einen sachlichen Bezugsrahmen für die nachstehende Literaturskizze zur Bewegung der Blockfreien abzugeben, soll zunächst einmal kurz der Problemhorizont des Phänomens Blockfreiheit umrissen werden.

\subsection{Problemhorizont der Blockfreiheit}

Blockfreiheit kann auf dreierlei Weise thematisiert werden: als ein System von Ideen bzw. als Ideologie, als außenpolitische Orientierung eines Staates und als eine kollektive, internationale Bewegung von Staaten. Dem letzteren Phänomen soll hier im folgenden besondere Aufmerksamkeit geschenkt werden.

In der Essenz ließe sich die Bewegung der Blockfreien als eine komplexe, vieldimensionale und multifunktionale Emanzipationsbewegung von Ländern bezeichnen, die nicht nur Objekte, sondern auch Subjekte der internationalen Beziehungen sein wollten: es waren allesamt Staaten, die entweder aufgrund ihrer erst frisch erlangten völkerrechtlichen Souveränität, ihrer militärischen Schwäche, ihrer politischen Instabilität oder ihrer sozio-ökonomischen Unterentwicklung innerhalb der Rangordnung des stratifizierten und bipolarisierten internationalen Systems gewissermaßen die "underdogs" darstellten. $\mathrm{Zu}$ verschiedenen Zeiten und in unterschiedlicher Intensität hatte die Bewegung für ihre Mitglieder drei wesentliche Bedeutungsinhalte: sie war eine Solidaritäts- und Protestbewegung zur Sicherung der nationalen Unabhängigkeit und zur Bekämpfung von Kolonialismus, Neo-Kolonialismus, Imperialismus, Rassismus und aller Formen von Fremdherrschaft und Fremdbestimmung; eine Defensivbewegung zur Reduzierung politischmilitärischer Spannungen im Ost-West-Konflikt; und sie war eine Bewegung zur Reform des Weltwirtschaftssystems bzw. eine internationale Interessengruppe zur Erlangung ökonomischer Vorteile im Nord-Süd-Konflikt. In den sechziger Jahren sahen die Blockfreien ihre Unabhängigkeit und den Weltfrieden vornehmlich durch den Ost-WestKonflikt und die Blockpolitik gefährdet; daher entschlossen sie sich zur Abstinenz von den Militärallianzen der Supermächte und zur Nichtidentifikation mit den Interessenlagen und Positionen der west-östlichen Streitparteien. In den siebziger Jahren jedoch betrieben die Blockfreien vordringlich ihre ökonomischen und entwicklungspolitischen Anliegen im Nord-Süd-Verhältnis und forderten eine Neue Weltwirtschaftsordnung und eine kollektive Self-Reliance der Dritten Welt gegenüber den Industrieländern.

Die Kernprobleme, denen sich die Bewegung der Blockfreien im Laufe ihrer Entwicklung ausgesetzt sah und weiterhin sieht, waren und sind im wesentlichen folgende:

- die Klärung des schwierigen Verhältnisses der Bewegung zu den Supermächten und Machtblöcken (Distanzfrage im Ost-West-Verhältnis, Zurückweisung des Postulats einer "Äquidistanz" zu den Blöcken, Verdeutlichung des Unterschiedes zwischen dem po-

4 Jayantanuja Bandyophadyaya, The Non-Aligned Movement and International Relations, in: India Quarterly (Neu-Delhi), vol. 33, 1977, S. 162. 
litischen (aktiven, dynamischen) Konzept der Blockfreiheit und dem juristischen (passiven, statischen) Konzept der Neutralität);

- die Erhaltung und Stärkung der Solidarität und Handlungsfähigkeit der Bewegung angesichts steigender Mitgliederzahlen und wachsender Heterogenität der Mitgliedschaft (demokratische Konsensfindung innerhalb der Bewegung, friedliche Streitbeilegung der Konflikte zwischen Mitgliedern, institutionell-organisatorische Ausdifferenzierung der Bewegung zur Verbesserung der Kommunikation, Kooperation und Koordination unter den Mitgliedern);

- die konzeptionelle Ausarbeitung und praktisch-politische Umsetzung der wichtigsten Anliegen der Bewegung in den Bereichen der Friedenssicherung (Abrüstung, Abbau der Machtblöcke, Entspannung und Friedenszonen), der Weltwirtschaftsreform (Neue Weltwirtschaftsordnung, Nord-Süd-Dialog), und der Süd-Süd-Kooperation (Aktionsprogramm für wirtschaftliche Zusammenarbeit, Kollektive Self-Reliance, Süd-Süd-Dia$\log )$.

\subsection{Informationsquellen zur Blockfreiheit}

Welche Informationsquellen stehen nun der Forschung über Blockfreiheit zur Verfügung? Willetts zufolge nicht sehr viele und verläßliche:

"Those who wish to study the Non-Aligned will find that very little research has been done on the Movement, much of the basic information is not readily available and most of the potential sources are not reliable. ${ }^{5}$

Knappe Hinweise auf die vorliegende Literatur und den Forschungsstand zur Blockfreiheit finden sich in Nord's Arbeit über Jugoslawien, in Willett's Dokumentation über die Gipfelkonferenz von Havanna und in der Chronik von Verlet. ${ }^{6}$ Als bibliographische Wegweiser lassen sich nennen die Bibliographie von Damian (Liste von Büchern in Englisch, Französisch, Deutsch und Russisch für den Zeitraum 1950-1975 sowie Liste von Zeitschriftenaufsätzen aus aller Welt für den Zeitraum 1970-1975), die Bibliographie von Singham/Tran Van Dinh (Liste ausgewählter Bücher und Zeitschriftenaufsätze ausschließlich in englischer Sprache) sowie eine Bibliographie des UN-Sekretariats.

Als wesentliches Hindernis für eine systematische Forschung über die Bewegung der Blockfreien galt bislang das Fehlen einer autoritativen, authentischen Dokumentensammlung. Die Blockfreien selbst haben eine umfassende Kodifizierung ihrer offiziellen Erörterungen und Entschließungen noch nicht in Angriff genommen, wenngleich auch eine wachsende Zahl von Dokumenten als UN-Dokumente klassifiziert wurden und zirkulierten und auch die Errichtung eines Blockfreien Dokumentations-Zentrums in Co-

5 Peter Willetts, The Non-Aligned in Havanna, London 1981, S. 71.

6 Lars Nord, Nonalignment and Socialism. Yugoslav Foreign Policy in Theory and Practice, Uppsala 1974, S. 2 ff.; Peter Willetts, a.a.O., S. 71 ff.; Martin Verlet, Le mouvement des non-alignés après La Havane: Contradictions et dynamique, in: Revue Tiers Monde (Chronique Internationale), t. XXI, no. 81, JanuarMärz 1980, S. 187. 
lombo/Sri Lanka projektiert wurde. ${ }^{7}$ Diesem Mangel ist nunmehr durch das monumentale, vierbändige Dokumentenwerk von Jankowitsch/Sauvant weitgehend abgeholfen worden: von der Kairoer Vorkonferenz (zum ersten Gipfel in Belgrad) vom Juni 1961 bis zum Herbst 1977 sind in dieser Dokumentation alle offiziellen Schlußdokumente der relevanten Blockfreien-Konferenzen enthalten; ferner eine Vielzahl sonstiger Dokumente (z. B. Papiere von Experten-Gruppen, Koordinierungs-Ländern, Hintergrundpapiere für das Koordinierungsbüro), während die Reden blockfreier Staatsmänner sowie Papiere einzelner Mitgliedsländer, die nicht von der Mehrheit der Mitglieder angenommen wurden, grundsätzlich nicht aufgenommen wurden. Neben der aktualisierenden Fortführung dieses Werkes hat Sauvant mittlerweile damit begonnen, ein analoges Werk für die "Gruppe der 77" herauszubringen.

\subsection{Forschungsansätze und Gesamtdarstellungen zur Blockfreiheit}

Versucht man einmal, die bisherigen Arbeiten zur Blockfreiheit systematisch zu kategorisieren, so ließen sich vielleicht je nach dem Fokus des Ansatzes (Analyse-Ebene, zeitliche und thematische Schwerpunktsetzung, theoretischer Anspruch) etwa die folgenden Unterscheidungen treffen:

- eine Gruppe von Arbeiten befaßt sich vor allem mit den historischen Wurzeln (AfroAsiatische Bewegung) und der Ideologie der Blockfreiheit (z. B. Jansen). Methodisch sind diese Studien meist historisch-deskriptiv angelegt. Als unergiebig erweist sich in dieser Gruppe das Bemühen um eine genaue Definition von Blockfreiheit, da es Lyon zufolge "at least as many serious working definitions of non-alignment" gibt "as there are members of the movement, and perhaps many more $"{ }^{8}$ Das Credo der Blockfreiheit läßt sich letztenendes nur über eine Auswertung der kumulierten offiziellen Dokumentation der Bewegung erschließen;

- eine zweite Gruppe von Arbeiten (meist aus den frühen sechziger Jahren) thematisiert Blockfreiheit vornehmlich im Kontext des Ost-West-Konflikts und versucht, theoretisch der "Realistischen Schule" der Lehre von den Internationalen Beziehungen verpflichtet, die blockfreie Politik mit Hilfe der klassischen Konzepte der Machtrivalität, der Allianzbildung und der Nationalinteressen zu erfassen (z. B. Martin). In dieser Perspektive erweist sich Blockfreiheit als eine Funktion des Ost-West-Konflikts, dient den ihr anhängenden Staaten zur Interessenmaximierung ("Schaukelpolitik") und entpuppt sich als ein "Trojanisches Pferd" des Kommunismus;

- eine weitere Gruppe von Arbeiten (aus den siebziger und achtziger Jahren) wiederum interessiert sich hingegen stärker für die Rolle der Blockfreiheit im Kontext des NordSüd-Konflikts. Diese Studien beschreiben und analysieren die ökonomischen und entwicklungspolitischen Konzepte und Aktivitäten der Blockfreien (Neue Weltwirtschafts-

7 Vgl. hierzu Third World Forum Newsletter, No. 4, Juni 1979, S. 9 und S. 40 ff.

8 Peter Lyon, Non-Alignment at the Summits. From Belgrade 1961 to Havana 1979-A Perspective View, in: Indian Journal of Political Science, vol. 41, No. 1, März 1980, S. 148. 
ordnung und Kollektive Self-Reliance), legen die Formierung der Bewegung der Blockfreien zu einer internationalen ökonomischen Interessengruppe dar und erörtern die Bedeutung der Blockfreien als einer Trägergruppe für verstärkte Süd-Süd-Kooperation (z. B. Sauvant);

- die wohl umfangreichste Gruppe von Arbeiten stellen Monographien zur Blockfreiheit als einer außenpolitischen Orientierung einzelner Länder (vor allem Jugoslawien und Indien) dar. Nord, der mit seiner Studie über Jugoslawien selber dieser Gruppe zuzurechnen ist, hat jedoch kritisiert, daß die meisten dieser Arbeiten zu stark deskriptiv oder affirmativ orientiert sind und zu wenig analytisch nach der Vermittlung von Theorie und Praxis blockfreier Außenpolitik fragen. ${ }^{9}$ Burton bemängelte im Hinblick auf diese Gruppe, daß "these studies are not satisfactory, for they do not distinguish those national influences which are unique to nonalignment, from others which are common to aligned alike"; ${ }^{10}$

- aus dieser Schwäche des monographischen Ansatzes leitete Burton die Notwendigkeit eines Ansatzes ab, "that can explain the occurrence of nonalignment in a similar form in a number of nations almost simultaneously, despite local circumstances which appear from national studies to be unique". ${ }^{11}$ Diesem Ansatz folgt nun eine letzte Gruppe von Arbeiten, die mit Gesamtdarstellungen und theoretischen Erklärungsversuchen Blockfreiheit als ein kollektives Phänomen internationaler Politik thematisieren und dieses Phänomen in eine allgemeine Theorie internationaler Beziehungen einzuordnen versuchen. Die interessantesten und anspruchsvollsten Erklärungsversuche haben in dieser Gruppe wohl Burton (Kommunikationstheorie), Korany (Situations-Rollen-Modell) und Willetts (quantitativer Forschungsansatz) unternommen. Im weiteren Umfeld einer theoretischen Verortung von Blockfreiheit in den internationalen Beziehungen scheinen ferner von Interesse zu sein das Studium der Außenpolitik von Entwicklungsländern (Clapham), das Konzept des "Kleinen Staates" (Vital) und dessen Lebensfähigkeit in der internationalen Politik sowie das Konzept der Schichtung und des Status in den internationalen Beziehungen (Lagos). ${ }^{12}$ Als Gesamtdarstellungen zur Blockfreiheit seien hier ferner erwähnt die Arbeiten von Mates, Singham und Colard.

Schon im Jahre 1965 erkannte Burton in dem Phänomen der Blockfreiheit eine Herausforderung für die Theorie Internationaler Beziehungen: "No theory of international relations is complete without an explanation of the development of, and a theory of, nonalignment. ${ }^{13}$ In kritischer Auseinandersetzung mit dem Macht-Modell der "Realistischen Schule" plädierte Burton für ein (alternatives) Kommunikations-Modell zur Analyse der Blockfreien-Bewegung:

9 Lars Nord, a.a.O., S. 4.

10 J. W. Burton, International Relations. A General Theory, Cambridge 1965, S. 165 f.

11 J. W. Burton, a.a.O., S. 167.

12 Christopher Clapham (ed.), Foreign policy making in developing states. A comparative approach, Westmead, Farnborough 1977; David Vital, The Inequality of States. A Study of the Small Power in International Relations, Oxf ord 1967; Gustavo Lagos, International Stratification and Underdeveloped Countries, Chapel Hill 1963.

13 J. W. Burton, a.a.O., S. 163. 
"In so far as the nonaligned States form a bloc or give expression to a community of interest, this is entirely on the basis of communication. The organizing pressures are not related to power...." Kommunikation war ihm zufolge "the only associative influence".

Dieses Kommunikations-Modell blieb lange Jahre verschüttet und wurde erst in späteren Jahren von dem indischen Forscher Bandyopadhyaya wieder aufgegriffen. ${ }^{14}$

Eine stark von persönlichen Erfahrungen geprägte Arbeit über die Blockfreien, die zugleich als eine Art von quasi-offizieller jugoslawischer Sicht der Dinge angesehen werden kann, legte im Jahre 1972 Mates vor, ehemaliger Sekretär der ersten Konferenz der Staats- und Regierungschefs blockfreier Länder in Belgrad und langjähriger Direktor des Belgrader Instituts für Politik und Wirtschaft. Als herausragende historische Entstehungsfaktoren für die Blockfreiheit nennt er einerseits "the loss of authority of the great powers and the destruction of the myth about their role as protectors of peace and order in the world" und andererseits "the breaking up of the colonial systems".

Eine ambitionierte Studie legte im Jahre 1976 der Ägypter Korany vor. Er strebt nichts weniger an als einen "systematic, holistic view of both the bases of third world foreign policy and of the structure and functioning of the non-aligned international subsystem" und beansprucht eine Forschungslücke zu füllen, da "no work has been published on the relationships among all non-aligned states analysed as an international subsystem". Mit großem Fleiß und großer intellektueller Anstrengung erarbeitete er "a great plum-pudding of a book", wie es ein angelsächsischer Kritiker etwas süffisant ausgedrückt hat. ${ }^{15}$ Seinen holistischen Erklärungsanspruch versucht er mit Hilfe eines Situations-RollenModells durch die Verschränkung dreier Analyse-Ebenen (internationales System, Gesellschaften der Dritten Welt und charismatische Führerpersönlichkeiten, exemplifiziert durch Fallstudien über Nasser und Nehru) einzulösen.

Ähnlich wie Burton weist auch Korany die Erklärungsrelevanz des klassischen MachtParadigmas der "Realisten" zurück, die Blockfreiheit nur im Ost-West-Zusammenhang zu deuten vermögen und tendenziell mit Neutralität gleichsetzen. Die reale Einwirkung blockfreier Politik auf das internationale System versucht er am Beispiel der "FriedensFunktion" der Blockfreien abzuschätzen, indem er die Effekte von Blockfreiheit auf Bipolarität, Multipolarität und Konflikt-Management untersucht.

Ähnlich wie Korany will auch Willetts mit seinem Buch aus dem Jahre 1978 eine Forschungslücke füllen. Ausgehend von dem Mangel an umfassenden analytischen Studien zur Blockfreiheit (im Vergleich zur Vielzahl deskriptiver Länder-Monographien) sieht er in seinem Werk "the first attempt to present a systematic profile of all the membership of the Non-Aligned ". Um die große Fülle empirischen Materials über Blockfreiheit sinnvoll verarbeiten zu können, bedient sich Willetts eines quantitativen Forschungsan-

14 Vgl. Jayantanuja Bandyophadyaya, The Non-Aligned Movement and International Relations, a.a.O. sowie ders., International Relations as a Communication System, in: International Studies, vol. 14, no. 2, April-Juni 1975.

15 Robert Purnell in: International Affairs, vol. 54, no. 2, April 1978, S. 296. 
satzes. Für die Jahre 1961 bis 1970 bemüht er sich (u. a. mit Hilfe einer Analyse des Abstimmungsverhaltens der Blockfreien in den Vereinten Nationen), Blockfreiheit als ein "distinct pattern of behavior" zu identifizieren. Brauchbare Abschnitte bietet das Buch auch zur Entstehung der Blockfreien-Bewegung und zu ihrer Institutionalisierung in den siebziger Jahren.

Im Jahre 1981 legte Willetts eine umfangreiche Dokumentation zu der spektakulären, scheinbar ganz im Zeichen der Ost-West-Rivalität stehenden Gipfelkonferenz der Blockfreien in Havanna vor. Neben einer sorgfältigen Edition der Konferenz-Dokumente enthält der Band einen längeren, einführenden theoretischen Essay, in dem der Autor sich kritisch mit der "realistischen Interpretation" der Havanna-Konferenz im OstWest-Kontext auseinandersetzt, die im Westen weit verbreitet ist. Ähnlich wie schon Burton und Korany weist auch Willetts diesen Erklärungsansatz explizit zurück und präsentiert eine alternative Erklärung der Konferenz, die von den Interessenlagen der Blockfreien selbst ausgeht und dementsprechend vor allem auch ökonomische und entwicklungspolitische Fragen der Bewegung in den Vordergrund rückt.

Erwähnenswert ist schließlich auch der von Singham edierte Sammelband zur Blockfreien-Bewegung in der Weltpolitik, der die Papiere einer Konferenz zur Blockfreiheit an der Howard University vom April 1976 vereint. Von den insgesamt 24 Beiträgen, die unter 5 Rubriken (Stellungnahmen von Politikern und Diplomaten zur Blockfreiheit, globale Massenkommunikation, nationale Befreiungsbewegung, Neue Weltwirtschaftsordnung und Afro-Amerikanische Reaktionen auf Blockfreiheit) abgehandelt werden, berührt jedoch nur ca. 1/4 unmittelbar Probleme der Blockfreiheit. Von Interesse ist der vom Herausgeber und verschiedenen Autoren vertretene polit-ökonomische Ansatz, der die materielle Basis und den strukturellen Rahmen des internationalen Systems zu erhellen bemüht ist, in dem die Bewegung der Blockfreien zu agieren hat.

\section{Phasen und Themen der Forschung über Blockfreiheit}

Entsprechend dem historischen Entwicklungsgang der Blockfreien-Bewegung und ihren jeweils vorherrschenden Themen könnte man, wenngleich auch etwas schematisch, verschiedene Phasen und Themen der Forschung über Blockfreiheit unterscheiden. In den sechziger Jahren standen Studien zur Blockfreiheit und der Afro-Asiatischen Bewegung sowie zur Blockfreiheit und dem Ost-West-Konflikt im Vordergrund, in den siebziger (und beginnenden achtziger) Jahren Untersuchungen zur Blockfreiheit und dem NordSüd-Konflikt.

\subsection{Blockfreiheit und Afro-Asiatische Bewegung}

Verschiedene Arbeiten widmen sich der Frage nach den historischen Ursprüngen der Idee und Bewegung der Blockfreiheit. Dabei werden weitgehend übereinstimmend deren Wurzeln im Kontext des Anti-Kolonialismus und Nationalismus zunächst in Süd- und Südostasien ausgemacht, der später im Zuge der Herausbildung der Afro-Asiatischen 
Solidaritätsbewegung auf Regionen Afrikas ausgedehnt wurde. Umstritten allerdings bleibt die Frage, ob die Bandung-Konferenz von 1955 als unmittelbarer Vorläufer der späteren Blockfreien-Konferenzen anzusehen ist. Vielfach wird hervorgehoben, daß die erste Gipfelkonferenz der Blockfreien in Belgrad 1961 aus der trilateralen Kooperation zwischen Ägypten (Nasser), Indien (Nehru) und Jugoslawien (Tito) in den Jahren zwischen 1956 und 1961 hervorging. ${ }^{16}$ Meist handelt es sich bei diesen Arbeiten um historisch-deskriptive Studien zu der afro-asiatischen Konferenz-Diplomatie aus europäischer Sicht (Smets, Guitard, Queille, Sasse, Schröder). Einen engagierten Beitrag aus der Sicht der afro-asiatischen Akteure legte der Inder Jansen vor. Der Sammelband von Sayegh bemüht sich um eine Typologie der Variationen von Blockfreiheit (in seinem Sprachgebrauch "Neutralismus") und erörtert die arabische Blockfreiheit (Fallstudien über Syrien, Algerien, Ägypten). Die Arbeit des Israeli Kimche ist die zeitlich weitreichendste Untersuchung über den Aufstieg und Niedergang der Afro-Asiatischen Bewegung und enthält Fallstudien über Indien, Indonesien und Tansania.

Eine kleinere Gruppe von Arbeiten konzentriert sich auf das Verhalten und die Gruppenformierungsprozesse afro-asiatischer (und anderer Entwicklungs-)Länder in den Vereinten Nationen (Pfahlberg, Kay, Stokman). Die Studie von Stokman scheint besonders interessant, weil sie differenziert die Formierung der späteren "Gruppe der 77" aus einer zunächst separaten afro-asiatischen und lateinamerikanischen Gruppierung aufzeigt.

\subsection{Blockfreiheit und Ost-West-Konflikt}

Eine Anzahl von Arbeiten aus den frühen sechziger Jahren (Martin, London, Lyon) reflektiert aus europäischer und amerikanischer Sicht die Besorgnisse des Westens, daß Blockfreiheit tendenziell zum Kommunismus neige und der Sowjetunion im Ost-WestKonflikt Zutritt zur Dritten Welt verschaffe. In dieser Perspektive war Blockfreiheit eine "opportunistische" Außenpolitik zur nationalen Interessenmaximierung (Ausspielen der Blöcke untereinander), die, wenn sie schon nicht eindeutig Partei ergreifen wollte, so doch zumindest im Ost-West-Konflikt strikte Neutralität (eine Einordnung der Blockfreiheit in die allgemeinen Dimensionen neutraler Politik unternahm Frei) wahren sollte. Eine löbliche Abweichung von dieser Perspektive stellt die gegenüber gängigen westlichen Vorurteilen und Stereotypen bezüglich der Blockfreiheit kritische Arbeit von Crabb dar (in Anlehnung an ein afrikanisches Sprichwort mit The Elephants and the Grass betitelt), die eine "sympathetic study of the neutralist credo" enthält. Im Kampf der "Elefanten" (= Super- und Blockmächte) ergreift Crabb eindeutig für das "Gras" (das dabei leidet) Partei (= Blockfreien). Zurückgewiesen wird die einseitige Verortung der Blockfreiheit im Ost-West-Konflikt auch in den Arbeiten von Burton, der insbesondere die Verwechslung von Blockfreiheit mit Neutralität kritisiert und dem Macht-Modell der "Realisten" ein Kommunikations-Modell zur Erklärung der Blockfreiheit ent- 
gegen setzt. Aus der Sicht der blockfreien Länder verteidigen die Inder Sen und Karunakaran die Abstinenz der Blockfreien von den Machtblöcken des Ost-West-Konflikts und machen die besonderen Interessen und Motive der Blockfreiheit abgehoben von diesem primär europäischen Konflikt deutlich.

\subsection{Blockfreiheit und Nord-Süd-Konflikt}

Die ökonomischen und entwicklungspolitischen Anliegen der Blockfreien wurden, obwohl sie bereits sehr frühzeitig (u. a. auf der Kairoer Konferenz der Entwicklungsländer zu Wirtschaftsfragen im Jahre 1962) zum Ausdruck kamen, von der Forschung erst recht spät in ihrer Bedeutung und Tragweite erkannt. Die beginnende internationale Debatte über die Errichtung einer Neuen Weltwirtschaftsordnung im Jahre 1974 setzte das Datum, von dem an die Forschung sich intensiv mit der ökonomischen Dimension von Blockfreiheit zu befassen begann. Auf der Suche nach den Ursprüngen des Konzepts der Neuen Weltwirtschaftsordnung leisteten hierbei die Studien von Jankowitsch und Sauvant Pionierarbeit. Sie wiesen darauf hin, daß unter dem Eindruck des Scheiterns westlicher Entwicklungsmodelle und -politiken die Blockfreien seit 1970 (Gipfelkonferenz von Lusaka) Wirtschafts- und Entwicklungsthemen zunehmende Bedeutung beimaßen und die Konzepte der kollektiven Self-Reliance und der Weltwirtschaftsreform erarbeiteten. Zugleich beobachteten sie im Zusammenhang mit der Abwendung der Blockfreien von Fragen der politischen Unabhängigkeit (im Ost-West-Konflikt) hin zu Fragen der wirtschaftlichen Unabhängigkeit (im Nord-Süd-Konflikt) eine Wandlung der Bewegung "von einem eher informellen Club gleichgesinnter Staats- und Regierungschefs zu einer hochorganisierten und -strukturierten internationalen "pressure group" mit dem Ziel der Neugestaltung des Weltwirtschaftssystems " ${ }^{17}$

Im gleichen Zusammenhang widmete sich Sauvant auch der Erforschung der "Gruppe der 77" und deren Verhältnis zu den Blockfreien, das er folgendermaßen umriß:

"Während die Blockfreien eine Schlüsselrolle bei dem Bemühen spielten, das Entwicklungsthema zu einem vorrangigen Punkt der internationalen Tagesordnung zu machen, ist die Gruppe der 77 zum Hauptorgan der Dritten Welt geworden, durch das die konkreten Änderungen . . . im Rahmen der UNO ausgehandelt werden. ${ }^{18}$

Mortimer erkennt in dieser Art arbeitsteiliger Zusammenarbeit von Blockfreien und Gruppe der 77 eine "Koalition der Dritten Welt", die seit 1974 den Industrieländern in internationalen Verhandlungen gegenübersteht und als Machtfaktor ernst genommen werden muß, wenngleich sie auch zunehmend brüchig werden könnte. Ähnlich sind auch für Berg und Silva die Blockfreien und die Gruppe der 77 zu den wichtigsten Instrumenten der Dritten Welt in deren Kampf um eine "Neue Weltordnung" geworden. In diesen Zusammenhängen sind auch die Forschungen über die UNCT AD von Bedeutung, deren

17 Karl P. Sauvant, Von der politischen zur wirtschaftlichen Unabhängigkeit? Die Ursprünge des Programms der Neuen Weltwirtschaftsordnung, in: Vereinte Nationen 2/79, S. 51.

18 Karl P. Sauvant, Die "Gruppe der 77"-Gewerkschaft der Dritten Welt, in: Vereinte Nationen 6/81, S. 191. 
Arbeit aus der Sicht der Dritten Welt Gosovic würdigt, während Rothstein sie (und die Forderungen der Entwicklungsländer) aus der Sicht der westlichen Industrieländer (namentlich der USA) scharf kritisiert.

\section{Forschung über Blockf reiheit in blockf reien Ländern}

Nennenswerte Forschung über Blockfreiheit findet, sieht man einmal von Jugoslawien und Indien ab, in den blockfreien Ländern selbst kaum statt. Doch weisen verschiedene internationale Tagungen auf ein gestiegenes Interesse an dieser Art von Forschung auch in anderen blockfreien Ländern hin.

Die quantitativ und qualitativ bedeutsamsten Arbeiten zur Blockfreiheit finden sich in Jugoslawien und Indien, zwei Gründungsländern der Bewegung der Blockfreien. In beiden Ländern kam und kommt, in Jugoslawien relativ stärker als in Indien, der Blockfreiheit eine eminent wichtige Bedeutung als außenpolitische Orientierung zu. In beiden Ländern wird auch der traditionelle Anspruch einer geistigen Führungsrolle in der Bewegung gepflegt. In Jugoslawien konzentriert sich die Forschung auf das Belgrader Institut für Politik und Wirtschaft und auf die Zeitschrift "Internationale Politik", die zugleich auch als das gewissermaßen internationale Sprachrohr für die offizielle jugoslawische Linie der Blockfreienpolitik gelten kann. Im Umfeld dieser beiden Institute wirkten und wirken Forscher wie Mates, Acimovic, Tadic und Petkovic.

In Indien konzentriert sich die Forschung über Blockfreiheit auf die School of International Studies der Jawaharlal University in New Delhi (mit der Zeitschrift "International Studies") und am Indian Council of World Affairs (mit den Publikationen "India Quarterly" und den "Foreign Affairs Reports"). Nach älteren zahlreichen Arbeiten vornehmlich über die indische Politik der Blockfreiheit sind jüngst zwei bemerkenswerte Sammelbände von Misra/Narayanan und Khan erschienen, die unter dem Eindruck der Gipfel-Konferenz von Havanna und der Afghanistan-Krise stehen und das mittlerweile breite Themen-Spektrum indischer Blockfreien-Forschung widerspiegeln.

Indische und jugoslawische Wissenschaftler sind auch maßgeblich an länderübergreifenden wissenschaftlichen Veranstaltungen zur Blockfreiheit beteiligt, wie sie in den letzten Jahren z. B. in New Delhi (indisch-jugoslawisches Symposium), in Lagos (Nigerianisches Institut für Internationale Beziehungen; erste wissenschaftliche Konferenz zur Blockfreiheit in Afrika mit Teilnehmern u. a. aus Nigeria, Indien, Sri Lanka, Sudan und Jugoslawien) und in Colombo (Bandaranaike-Zentrum für Internationale Studien mit Wissenschaftlern, Diplomaten und Journalisten aus Bangladesh, Kuba, Ägypten, Indien, Jugoslawien, Sambia, Pakistan und Sri Lanka) stattfanden. ${ }^{19}$

19 Vgl. hierzu Internationale Politik (Belgrad), H. 717, 20. Februar 1980, S. 24 f.; H. 724, 5. Juni 1980, S. 10 ff.; H. 740 , 5. Februar 1981 , S. $21 \mathrm{ff}$. 


\section{Blockfreiheit: Zukunftsperspektiven und Forschungsdesiderata}

Trotz relativ großer innerer und äußerer Belastungen (u. a. Konflikte unter Blockfreien, Afghanistan-Krise) wird die Bewegung der Blockfreien nicht zerfallen oder an Bedeutung verlieren, sondern sich konsolidieren und weiterentwickeln. Weder sind die historischen Gründe für ihre Existenz entfallen noch hat sie ihre Emanzipationsziele erreicht. Unter den Gruppierungen der Dritten Welt weisen die Blockfreien auch weiterhin die aktivste Mitgliedschaft, die größte thematische Spannweite, das höchste Prestige und den gewichtigsten Einfluß auf. Neben der Stärkung der Einheit der Bewegung durch demokratischen Streitaustrag und friedliche Konfliktregelung wird es zu den Aufgaben der achtziger Jahre gehören, den wiederbelebten Ost-West-Konflikt zu entspannen und den Nord-Süd-Dialog erneut zu dynamisieren, vor allem durch einen konsequenten Ausbau der kollektiven Self-Reliance.

Für die.Forschung über Blockfreiheit könnten sich aus dieser Einschätzung etwa die folgenden Schwerpunkte ergeben. Zum einen sollte sie verstärkt nach der möglichen "Friedens-Funktion" (Korany) der Bewegung der Blockfreien fragen. Hier wäre sowohl die klassische "Distanzfrage" aufzugreifen und das Verhältnis der Blockfreien zu den Blockmächten, insbesondere zur UdSSR (ein bisher stark unterbelichtetes Thema), ${ }^{20} \mathrm{zu}$ bearbeiten, als auch vor allem an die Forschungen von Bebler und Jütte über Sicherheitsaspekte von Blockfreiheit und das Verhältnis von Blockfreiheit und Abrüstung anzuknüpfen. ${ }^{21}$ Erweitert werden müßte die Forschung in diesem Bereich um Analysen der Implikationen intra-blockfreier kriegerischer Konflikte für die Politik der Blockfreiheit. Neue Bedeutung haben mit der aufkommenden Friedensbewegung und -diskussion in Europa auch Uberlegungen bezüglich eines engeren Zusammengehens der Blockfreien mit den klassischen europäischen Neutralen und anderen interessierten Staaten bei der Konzipierung von alternativen Sicherheitspolitiken im Bereich der west-östlichen Paktsysteme erhalten. Bemerkenswert ist, daß in diesen letzteren Zusammenhängen auch die internationale Friedensforschung die Blockfreien gewissermaßen "neu entdeckt" hat. ${ }^{22}$ Theoretisch angeknüpft werden könnte in diesem Forschungsbereich an das Kommunikations-Paradigma von Burton und Bandyopadhyaya, das dem klassischen Macht-Paradigma der "Realisten" entgegengestellt werden müßte und das ein "futuristisches Modell" internationaler Beziehungen entwirft, in dem Macht durch Kommunikation ersetzt wird, Kooperation statt Konfrontation vorherrscht, und Blockfreiheit als einen neuen

20 Klaus Fritsche, Die Bewegung Blockfreier Staaten in Sowjetischer Sicht, Arbeitspapier der "Dokumentationsstelle Bewegung Blockfreier Staaten, e. V.", Dortmund, Februar 1982, S. 2, hält es für erstaunlich, "daß ausführliche Untersuchungen über das Verhältnis der Sowjetunion zur Blockfreienbewegung nicht existieren, und auch in der sowjetischen Forschung die ... Politik der Nichtpaktgebundenheit nicht vollständig und gründlich genug ... untersucht" wurde.

21 Anton Bebler, Security Aspects of Non-Alignment, in: International Studies (New Delhi), vol. XIV, AprilJuni 1975, S. 289-302; Rüdiger Jütte, Blockfreiheit und Abrüstung, in: Internatoinale Entwicklung (Wien), 1981/II, S. 3-21.

22 Vgl. hierzu die Konferenzberichte von Hans Günter Brauch und Eva Senghaas-Knobloch in: DGFK-Informationen $2 / 81$, S. $42-47$. 
"Verhaltens-Standard" zwischenstaatlicher Beziehungen propagiert, der die politischen Verkehrsformen der klassischen Staatenwelt (Macht-, Allianz- und Hegemonialpolitik zur Sicherung der Nationalinteressen) langfristig ablösen soll.

Zum anderen wäre die Forschung auf die Frage zu konzentrieren, auf welche Weise und in welchen Bereichen und mit welchen Mitteln die Blockfreien ihre Zusammenarbeit untereinander (und mit anderen Entwicklungsländern) verstärken und (in enger Zusammenarbeit mit der Gruppe der 77 und anderen Gruppierungen der Dritten Welt) die SüdSüd-Kooperation insgesamt intensivieren könnten. Hierbei ginge es vor allem um eine Abschätzung der Realisierungschancen des Konzepts und der Programmatik kollektiver Self-Reliance, sowohl im Sinne verstärkter Verhandlungsmacht zur Herbeiführung einer Neuen Weltwirtschaftsordnung als auch im Sinne alternativer Strategien zur Uberwindung von Unterentwicklung. Anzuknüpf en wäre in diesem Forschungsbereich namentlich an die Arbeiten von Sauvant. ${ }^{23}$

\section{Verzeichnis der erwähnten und kommentierten Literatur}

\section{Bibliographien}

Damian, A., Bibliography of Selected Books and Articles on Nonalignment, Institute of International Politics and Economics, Beograd 1975

Singham, A. W./Tran Van Dinh Conferences of the Non-Aligned Countries 1955-75 (edited with Commentary), New York, o. J.

United Nations, Secretariat, Non-Alignment. A Select Bibliography, New York, ST/LIB/SER, B/18, 15 July 1975

\section{Dokumentensammlungen}

Jankowitsch, O./Sauvant, K.P., The Third World Without Superpowers: The Collected Documents of the NonAligned Countries (Vol. I-IV), Dobbs Ferry, New York 1978

Sauvant, K. P., The Third World Without Superpowers. 2nd Series. The Collected Documents of the Group of 77 (Vol. I ff.), Dobbs Ferry, New York 1981

23 Vgl. z. B. Karl P. Sauvant, Organizational Infrastructure for Self-Reliance: The Non-Aligned Countries and the Group of 77, Arbeitspapier für die "International Conference on the Principles of Non-Alignment" in Bagdad (4.-6. Mai 1982), New York, Januar 1982; Annemarie Grosse-Jütte/Rüdiger Jütte, Neue Weltwirtschaftsordnung und Self-Reliance: Konzeptionelle Beiträge und Institutionelle Entwicklung der Blockfreien Bewegung, Arbeitspapier für den Kongreß der Deutschen Vereinigung für Politische Wissenschaft in Augsburg 1979 (Spezialistentreffen: AK Internationale Organisation \& Integration); Irena Reuter-Hendrichs, Jugoslawiens Wirtschaftsbeziehungen zu den Staaten der Dritten Welt im Zusammenhang seiner Interessen an und in der Blockfreien-Bewegung, Stiftung Wissenschaft und Politik, Ebenhausen, Januar 1982; Anselm Skuhra, Die entwicklungspolitische Konzeption der Blockfreien, in: epd-Entwicklungspolitik 7/81 (Die Blockfreien), S. 13-17; Volker Matthies (Hrsg.), Süd-Süd-Beziehungen. Zur Kommunikation, Kooperation und Solidarität zwischen Entwicklungsländern, München-Köln-London 1982 (i. V.). 
Arbeiten zur Afro-Asiatischen Bewegung, zu Ost-West-Fragen und erste Erklärungsversuche der Blockfreiheit

Acimovic, L. (ed.), Non-Alignment in the World of Today, Belgrad 1969

Burton, J. $W$., International Relations. A General Theory, Cambridge 1965

Ders. (ed.), Non-Alignment, London 1966

Crabb, C. V., The Elephants and the Grass: A Study of Nonalignment, New York 1965

Frei, D., Dimensionen neutraler Politik. Ein Beitrag zur Theorie der Internationalen Beziehungen, Genf 1969

Guitard, O., Bandoung et le réveil des anciens colonisés, Paris 1965

Jansen, G. H., Afro-Asia and Non-Alignment, London 1966

Karunakaran, K. P., Outside the Contest. A Study of Nonalignment, New Delhi 1963

Kay, D. A., The New Nations in the United Nations: 1960-1967, New York 1970

Kimche, D., The Afro-Asian Movement: Ideology and Foreign Policy of the Third World, Jerusalem 1973

London, $K$. (ed.), New Nations in a Divided World. The International Relations of the Afro-Asian States, New York-London 1963

Lyon, $P$., Neutralism, Leicester 1963

Martin, L. W. (ed.), Neutralism and Nonalignment. The New States in World Affairs, New York 1962

Pfahlberg, B., Zur Politik afro-asiatischer Staaten in den Vereinten Nationen 1945-1960, Baden-Baden 1966

Queille, $P$., Histoire de l'Afro-Asiatisme jusquà Bandoung, Paris 1965

Sasse, $H$., Die asiatisch-afrikanischen Staaten auf der Bandung-Konferenz, Frankfurt am Main und Berlin 1958

Sayegh, F. A. (ed.), The Dynamics of Neutralism in the Arab World, San Francisco 1964

Schröder, D., Die Konferenzen der "Dritten Welt", Hamburg 1968

Sen, B., Against the Cold War, Bombay 1962

Smets, $P$. F., De Bandoung à Moshi: contribution à l'étude des conférences afro-asiatiques, 1955-1963, Brüssel 1964

Stokman, F. N., Third World Group Formation in the United Nations, Amsterdam 1977

\section{Arbeiten zu Nord-Süd-Fragen, Gesamtdarstellungen und theoretische Erklärungsversu- che}

Berg, E., Non alignement et nouvel ordre mondial, Paris 1980

Colard, D., Le Mouvement des Pays Non-Alignés, Paris 1981

Gosovic, B., UNCTAD: Conflict and Compromise. The Third World's Quest for an Equitable World Economic Order through the United Nations, Leiden 1972

Khan, $R$. (ed.), Perspectives on Non-Alignment, New Delhi 1981

Korany, B., Social Change, Charisma and International Behaviour: Toward a Theory of Foreign Policy-making in the Third World, Leiden 1976

Mates, L., Nonalignment: Theory and Current Policy, Dobbs Ferry-New York 1972

Misra, K.P./K. R. Narayanan (eds.), Non-Alignment in Contemporary International Relations, New Delhi 1981

Mortimer, R. A., The Third World Coalition in International Politics, New York 1980

Rothstein, R. L., The Weak in the World of the Strong: The Developing Countries in the International System, New York 1977

Ders., Global Bargaining: UNCTAD and the Quest for a New International Economic Order, Princeton 1979

Sauvant, K. P./O. Jankowitsch, The Initiating Role of the Non-Aligned Countries, in: Sauvant, K. P. (ed.), Changing Priorities on the International Agenda. The New International Economic Order, Oxf ord usw. 1981

Sauvant, K. P., The Origins of the NIEO Discussions, in: Ders. (ed.) a.a.O.

Silva, Leelananda de. The Non-Aligned Movement and the Group of 77: Third World Instruments for a New World Order, Genf 1981

Singham, A. W. (ed.), The Non-Aligned Movement in World Politics, Westport, Conn. 1977

Willetts, $P$., The Non-Aligned Movement. The Origins of a Third World Alliance, London 1978

Ders., The Non-Aligned in Havanna, London 1981 


\title{
The Movement of Non-Alignment
}

\author{
by Volker Matthies
}

Those who wish to study the Non-Aligned will find that very little research has been done on the Movement, much of the basic information is not readily available and most of the potential sources are not reliable. This article tries to give some hints at sources of information on the Non-Aligned Movement.

Most of the determinants of Non-Alignment were rooted in colonial history, in the process of de-colonization, and in the social, political and economic situation of the new states. The rapid escalation of the Cold War added a new urgency to the policy of NonAlignment and reinforced the factors which provided its rationale. But Non-Alignment was not caused by the Cold War, nor was it dependent on the Cold War for its survival. In the course of the rapid development as an international movement, Non-Alignment has changed its character and content in an important aspect. From a primarily political strategy it has transformed itself into a collective economic movement of the Third World countries and spearheaded the demand and the struggle for a new international economic order. 